Pacific Journal of Mathematics

DIFFERENTIAL SIMPLICITY AND EXTENSIONS OF A 


\title{
DIFFERENTIAL SIMPLICITY AND EXTENSIONS OF A DERIVATION
}

\author{
Yves LeqUAIN
}

\begin{abstract}
Let $R$ be an integral domain containing the rational numbers, $K$ its quotient field and $\Omega$ an algebraic closure of $K$; let $D$ be a derivation on $R$ such that $R$ is $D$-simple. The valuation rings $V$ such that $R \cong V \cong \Omega$ on which $D$ is regular are determined.
\end{abstract}

Introduction. Let $R^{\prime}$ be the complete integral closure of $R$ in $K$. Seidenberg has shown that $D$ is regular on $R^{\prime}$ [3]. We want here to continue his work and determine all the valuation rings $V$ such that $R \subseteq V \subseteq \Omega$ on which $D$ is regular.

First we determine in paragraph 2 the valuation rings of $K$ that have property, and we show that they are in 1-1 correspondence with the proper prime ideals of $R$.

Then, in paragraph 4 we show that if $V$ is a valuation ring such that $R \cong V \subseteq \Omega$, then $D$ is regular on $V$ if and only if $V$ is unramified over $K$ and $D$ is regular on $V \cap K$. To do that, we have to show first in paragraph 3 that if $B$ is a valuation ring of $\Omega$ such that $B \cap$ $K$ is rank-1 discrete and contains the rational numbers, then its inertia field over $K$ can be obtained as the intersection of a formal power series field with $\Omega$.

1. Preliminaries. Let $R$ be a commutative ring with identity. A derivation $D$ of $R$ is a map from $R$ into $R$ such that $D(a+b)=$ $D(a)+D(b)$ and $D(a b)=a D(b)+b D(a)$ for all $a, b \in R$. An ideal $I$ of $R$ is a $D$-ideal if $D(I) \subseteq I ; R$ is $D$-simple if it has no $D$-ideal other than (0) and (1). If $R$ is a $D$-simple ring of characteristic $p \neq 0, R$ is a primary ring [2, Theorem 1.4], hence is equal to its total quotient ring; this case will not be of interest in our considerations.

Thus, let $R$ be a $D$-simple ring of characteristic 0 , which is then a domain containing the rational numbers [2, Corollary 1.5]; let $K$ be its quotient field and $\Omega$ an algebraic closure of $K$. The derivation $D$ can be uniquely extended to a derivation of $\Omega$, which we also call $D$, and if $N$ is any field between $K$ and $\Omega$, we have $D(N) \subseteq N$ [6, Corollary 2', p. 125]. If $S$ is a ring with quotient field $N$ such that $D(S) \subseteq$ $S$, we shall say that $D$ is regular on $S$, or that $(N, S)$ is $D$-regular, or that $D$ can be extended to $S$.

We note that if $D$ is regular on a ring $S$ and if $M$ is a multiplicative system of $S$, then $D$ is regular on $S_{M}$. We note also that if $R$ is $D$-simple, and if $S$ is a ring such that $R \subseteq S \subseteq \Omega$, then to say that 
$D$ is regular on $S$ is equivalent to saying that $S$ is $D$-simple, indeed:

Proposition 1.1. Let $R$ be a D-simple ring with quotient field $K$; let $\Omega$ be an algebraic closure of $K$, and $S$ a ring such that $R \subseteq$ $S \subseteq \Omega$. If $D$ is regular on $S$, then $S$ is $D$-simple.

Proof. It will be enough to show that if $I$ is a nonzero ideal of $S$, then $I \cap R$ is a nonzero ideal of $R$. Let $0 \neq x \in I$, and let $X^{n}+$ $k_{1} X^{n-1}+\cdots+k_{n} \in K[X]$ be its minimal polynomial over $K$ where we note that $k_{n} \neq 0$; then, from the equality $x^{n}+k_{1} x^{n-1}+\cdots+k_{n}=0$, we can get $r_{0} x^{n}+r_{1} x^{n-1}+\cdots+r_{n}=0$ with $r_{i} \in R \subseteq S$ for $i=0,1, \cdots, n$, and $r_{n} \neq 0$, so that we have $0 \neq-r_{n}=r_{0} x^{n}+r_{1} x^{n-1}+\cdots+r_{n-1} x \in I \cap R$.

Let $L$ be a field, $N$ an algebraic extension of $L$, and $V$ a valuation ring of $N$. We shall denote the inertia degree of $V$ over $L$ by $f(V \mid L)$, and the ramification index of $V$ over $L$ by $e(V \mid L)$. If $A$ is a valuation ring of $L$, following Endler's terminology in [1], we shall say that $A$ is indecomposed in $N$ if there is only one valuation ring of $N$ lying over $A$, and, when $N$ is a finite extension of $L$, we shall say that $A$ is defectless in $N$ if $[N: L]=\sum_{i=1}^{m} e\left(V_{i} \mid L\right) f\left(V_{i} \mid L\right)$ where $\left\{V_{1}, \cdots, V_{m}\right\}$ is the set of valuation rings of $N$ lying over $A$.

An ideal $I$ of a ring $S$ will be said to be proper if it is different from $S$. We shall use $D^{(0)}(x)$ to denote $x$, and for $n \geqq 1, D^{(n)}(x)$ to denote $D\left(D^{(n-1)}(x)\right)$, i.e., the $n$th derivative of $x$.

2. Extensions of the derivation in the quotient field.

Lemma 2.1. Let $R$ be a ring, $D$ a derivation on $R, P$ a prime ideal of $R$ containing no $D$-ideal other than (0). Define $v: R \backslash\{0\} \rightarrow$ $\{$ nonnegative integers $\}$ by $v(x)=n$ if $D^{(i)}(x) \in P$ for $i=0, \cdots, n-1$ and $D^{(n)}(x) \notin P$. Then,

(i) $R$ is a domain.

(ii) $v$ is the trivial valuation if $P=(0)$, and is a rank-1 discrete valuation if $P \neq(0)$.

(iii) The valuation ring $R_{v}$ of $v$ contains $R$, and its maximal ideal $\mathfrak{M}_{v}$ lies over $P$.

Proof. See [2, Theorem 3.1]. Note that for $x \in R \backslash\{0\}$ we indeed have $v(x)<\infty$ for otherwise the ideal generated by $\bigcup_{i=0}^{\infty} D^{(i)}(x)$ would be a nonzero $D$-ideal contained in $P$, which cannot be. Note also that the property for $P$ to contain no $D$-ideal other than (0) is equivalent to $R_{P}$ being $D$-simple.

Lemma 2.2. Let $R, D, P, v, R_{v}, \mathfrak{M}_{v}$ be as in 2.1. Let $K$ be the 
quotient field of $R$. Let $S$ be a ring between $R$ and $K$ such that $D$ is regular on $S$. Then, the following statements are equivalent:

(i) $S \subseteq R_{v}$.

(ii) There is a prime ideal $Q$ of $S$ lying over $P$.

In this case, $Q$ is the only prime ideal of $S$ lying over $P$ and is equal to $\mathfrak{M}_{v} \cap S$.

Proof. If $S \subseteq R_{v}$, take $Q=\mathfrak{M}_{v} \cap S$. Conversely, suppose there exists a prime ideal $Q$ of $S$ such that $Q \cap R=P$. Being regular on $S, D$ is also regular on $S_{Q}$; furthermore, $S_{Q} \supseteqq R_{P}$, and $R_{P}$ is $D$-simple, thus by $1.1 S_{Q}$ is $D$-simple. Then, by 2.1 , we can define a valuation $w: S \backslash\{0\} \rightarrow$ nonnegative integers $\}$ by $w(y)=m$ if $D^{(j)}(y) \in Q$ for $j=$ $0, \cdots, m-1$ and $D^{(m)}(y) \notin Q$; calling $S_{w}$ the valuation ring of $w$, we have $S \subseteq S_{w}$. At the same time, we will have the valuation $v$ defined with the prime ideal $P$ of $R$, and for an element $x \in R \backslash\{0\}$ we have $D^{(i)}(x) \in P$ if and only if $D^{(i)}(x) \in Q$ since $P=Q \cap R$; thus, $v=w$ on $R$, hence also $v=w$ on $K$, and $S \subseteq S_{w}=R_{v}$. Furthermore, by 2.1, we have $Q=\mathfrak{M}_{w} \cap S$, hence also $Q=\mathfrak{M}_{v} \cap S$, so that $\mathfrak{M}_{v} \cap S$ is the unique prime ideal of $S$ lying over $P$.

Lemma 2.3. Let $A$ be a D-simple valuation ring. Then, $A$ is a field or is a rank-1 discrete valuation ring.

Proof. If $A$ is not a field, and $\mathfrak{U} \neq(1)$ is any ideal of $A$, then $\bigcap_{n=0}^{\infty} \mathfrak{U}^{n} \neq(1)$ is a $D$-ideal; thus, $A$ being $D$-simple, we have $\bigcap_{n=0}^{\infty} \mathfrak{A}^{n}=$ (0) and $S$ is a rank-1 discrete valuation ring.

TheOREM 2.4. Let $R$ be a D-simple ring with quotient field $K$. Let $\mathscr{P}=\{$ proper prime ideals of $R\}$, and $\mathscr{V}=$ valuation rings of $K$ containing $R$ to which $D$ can be extended\}. Define $\varphi: \mathscr{P} \rightarrow \mathscr{C}$ by $\varphi(P)=R_{v}$ where $v$ is the valuation associated to $P$ by 2.1. Then, $\varphi$ is a bijection.

Proof. Let us show first that $D$ is regular on $R_{v}$. Let $a b^{-1}$ be any element of $R_{v}$ with $a, b \in R, b \neq 0, v(a) \geqq v(b)$; then $D\left(a b^{-1}\right)=$ $[b D(a)-a D(b)] b^{-2}$. If $v(a)>v(b)$, then $v(D(a))=v(a)-1 \geqq v(b)$ and $v(D(b)) \geqq v(b)-1$, so that $v(b D(a)-a D(b)) \geqq \inf \{v(b)+v(D(a)), v(a)+$ $v(D(b))\} \geqq 2 v(b)$ and $D\left(a b^{-1}\right) \in R_{v}$. If $v(a)=v(b)=0$, then $v(b D(a)-$ $a D(b)) \geqq 0=2 v(b)$ and $D\left(a b^{-1}\right) \in R_{v}$. If $v(a)=v(b)=n>0$, then $v(b D(a))=$ $v(a D(b))=2 n-1$ so that $v(b D(a)-a D(b)) \geqq 2 n-1$; furthermore we have $D^{(2 n-1)}(b D(a))=\sum_{i=0}^{2 n-1} C_{2 n-1}^{i} D^{(i)}(b) D^{(2 n-i)}(a)=\alpha_{1}+C_{2 n-1}^{n} D^{(n)}(b) D^{(n)}(a)$ with $\alpha_{1} \in P$, and similarly $D^{(2 n-1)}(a D(b))=\alpha_{2}+C_{2 n-1}^{n} D^{(n)}(a) D^{(n)}(b)$ with $\alpha_{2} \in P$, so that $D^{(2 n-1)}(b D(a)-a D(b))=\alpha_{1}-\alpha_{2} \in P$; hence $v(b D(a)-$ 
$a D(b)) \geqq 2 n$ and $D\left(a b^{-1}\right) \in R_{v}$. Thus, $D$ is regular on $R_{v}$.

If $\mathfrak{M}_{v}$ is the maximal ideal of $R_{v}$, we have $P=\mathfrak{M}_{v} \cap R$ by 2.1, thus $\varphi$ is injective.

Now, let $A$ be a valuation ring of $K$ containing $R$ to which $D$ can be extended. If $A=K$, we clearly have $A=\varphi((0))$. If $A \neq K$, let $Q$ be its maximal ideal. Let $P=Q \cap R$, let $v$ be the valuation associated to $P$ by 2.1 , and let $R_{v}$ be the valuation ring of $v$. Since $P$ is different from (0), $R_{v}$ is different from $K$; by 2.2 , we have $A \subseteq$ $R_{v}$; by $1.1 A$ is $D$-simple, and hence has rank-1 by 2.3. Thus $A=$ $R_{v}, A=\varphi(Q \cap R)$, and $\varphi$ is surjective.

CoRollary 2.5. Let $R$ be a $D$-simple ring with quotient field $K$. Let $A$ be a valuation ring of $K$ which contains $R, Q$ its maximal ideal, $P$ its center over $R$, and $v$ the valuation associated to $P$ by 2.1 . Then, the following statements are equivalent:

(i) $D$ can be extended to $A$.

(ii) For any $a, b \in P$ such that $v(a) \geqq v(b)$, then $a b^{-1} \in A$.

(iii) For any $x \in A$, there exists $a, b \in R$, such that $x=a / b$ and $v(a) \geqq v(b)$.

Remember that for an element a of $R, v(a)$ is the number of successive applications of the derivation $D$ necessary to get a out of the center $P$.

Proof. The condition (ii) is equivalent to $R_{v} \subseteq A$; the condition (iii) is equivalent to $A \subseteq R_{v}$. But in both cases $A$ and $R_{v}$ have the same center on $R$; thus, both conditions (ii) and (iii) are equivalent to $A=R_{v}$, i.e., equivalent to (i).

3. On the inertia field. Let $N$ be a normal algebraic extension of $K$ (possibly infinite), and $G$ its Galois group. Let $B$ be a valuation ring of $N, \mathfrak{M}_{B}$ its maximal ideal; let $\pi$ be a place of $N$ corresponding to $B$ and $\mu$ its residue field; let $v$ be a valuation of $N$ corresponding to $B$ and $\Delta$ its value group. Let $A=B \cap K, A$ its residue field and $\Gamma$ its value group; $\mu$ is a normal algebraic extension of $A[1,(14.5)]$. The inertia group of $B$ over $K$ is $G^{T}(B \mid K)=$ $\left\{\sigma \in G / \sigma x-x \in \mathfrak{M}_{B} \forall x \in B\right\}=\{\sigma \in G / \pi \circ \sigma=\pi\}$; it is a closed subgroup of $G$ [1, (19.2)]; its fixed field $K^{T}(B \mid K)=\left\{y \in N / \sigma y=y \forall \sigma \in G^{T}(B / K)\right.$ is the inertia field of $B$ over $K$.

In this section, we shall only be concerned with the case of $A=$ $B \cap K$ being a rank-1 discrete valuation ring which contains the rational numbers. Note that $B$ has to be of rank-1 too [1, (13.14)]. We have: 
Proposition 3.1. $K^{T}(B / K)$ is the smallest field $L$ between $K$ and $N$ such that $B \cap L$ is indecomposed in $N$ and such that $\mu$ is purely inseparable over the residue field $\Lambda^{L}$ of $B \cap L$.

Proof. See [1, (19.11)].

Proposition 3.2. $K^{T}(B \mid K)$ is the unique field $L$ between $K$ and $N$ such that $B \cap L$ is indecomposed in $N, f(B \mid L)=1$ and $e(B \cap L \mid K)=$ 1.

Proof. Since $A$ contains the rational numbers, $A$ has characteristic zero, $\mu$ is a separable extension of $\Lambda$, and, by 3.1, $K^{T}(B \mid K)$ is the smallest field $L$ between $K$ and $N$ such that $B \cap L$ is indecomposed in $N$ and $f(B \mid L)=1$. Now, $N$ is also separable over $K$ so that $\Gamma^{T}=\Gamma$, and $B \cap K^{T}(B \mid K)$ is a rank-1 discrete valution ring; then $B \cap K^{T}(B \mid K)$ is defectless in all the finite extensions of $K^{T}(B \mid K)$ contained in $N$ [6, Corollary, p. 287], and $K^{T}(B \mid K)$ is maximal among the fields $L$ that have the property $f(B \mid L)=1$ and $e(B \cap L \mid K)=1$.

Proposition 3.3. $K^{T}(B \mid K)$ is the biggest field $L$ between $K$ and $N$ such that $e(B \cap L \mid K)=1$.

Proof. Let $L$ be a field between $K$ and $N$ such that $e(B \cap L \mid K)=$ 1. Let $L^{T}(B \mid L)$ be the inertia field of $B$ over $L$; by $3.2, B \cap L^{T}(B / L)$ is indecomposed in $N, f\left(B \mid L^{T}(B \mid L)\right)=1$ and $e\left(B \cap L^{T}(B \mid L) \mid L\right)=1$, hence also $e\left(B \cap L^{T}(B \mid L) \mid K\right)=1$ since $e(B \cap L \mid K)=1$. Thus, by 3.2, $L^{T}(B \mid L)=K^{T}(B \mid K)$ and $L \subseteq K^{T}(B \mid K)$.

COROLlaRY 3.4. Let $V$ be a valuation ring contained in $N$ lying over $A$. Then, the following statements are equivalent:

(i) $e(V / K)=1$.

(ii) There exists a valuation ring $E$ of $N$ lying over $V$ such that $V \subseteq K^{T}(E / K)$.

(iii) For every valuationring $E$ of $N$ lying over $V, V \cong K^{T}(E / K)$.

Now, let $\left(N^{*}, B^{*}\right)$ be a completion of $(N, B)$; by this, we mean that $N^{*}$ is a $B$-completion of $N\left[5,(1-7-1)\right.$, p. 27], and $B^{*}$ the topological closure of $B$ in $N^{*}$; let $\left(K^{*}, A^{*}\right)$ be the completion of $(K, A)$ contained in $\left(N^{*}, B^{*}\right)$. $A$ being a rank-1 discrete valuation ring, we let $t$ be a generator of the maximal ideal of $A$. Let $\sigma^{\prime}$ be an algebraic closure of $N^{*}$, and $\delta$ the algebraic closure of $K^{*}$ contained in $\sigma^{\prime \prime}$. Let $C$ be a field of representatives of $A^{*}$ and $\bar{C}$ the algebraic closure of $C$ contained in $\delta$; by [7, Theorem 27, p. 304], we have $A^{*}=C[[t]]$ and $K^{*}=C((t))$. Let $F$ be the unique valuation ring of $\tilde{\sigma}$ which lies over $A^{*}[5,(2-1-3)$, p. 44]. The situation can be resumed by the fol- 
lowing diagram:

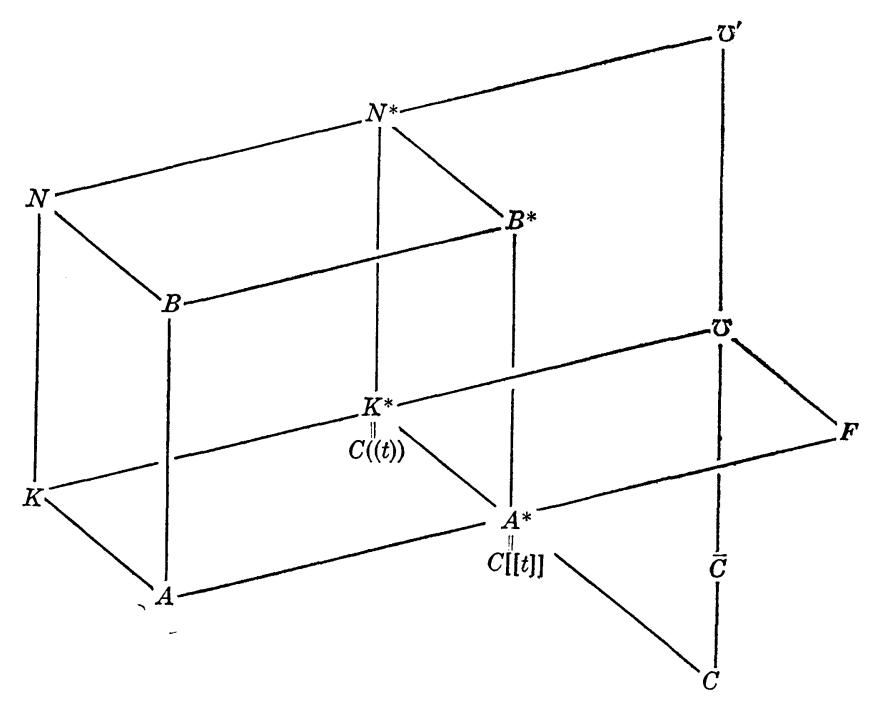

Proposition 3.5. $K^{T}(B / K)=\bar{C}((t)) \cap N$ and $B \cap K^{T}(B / K)=\bar{C}[[t]] \cap$ $N$.

Proof. We shall do it in several steps.

Step 1. $\bar{C}((t)) \cap \tilde{O}$ is the inertia field of $F$ over $K^{*}$ and $\bar{C}[[t]] \cap$ $\ddot{\sigma}=F \cap(\bar{C}((t)) \cap \widetilde{C})$.

Proof. $\quad \bar{C}[[t]] \cap \tilde{O}$ is a valuation ring of $\bar{C}((t)) \cap \tilde{O}$ which lies over $A^{*}=C[[t]]$; thus it is indecomposed in $\widetilde{O}$ and is equal to $F \cap(\bar{C}((t)) \cap \tilde{O})$. Let $\xi$ (respectively $w$-) be a place (respectively a valuation) of $\tilde{O}$ corresponding to the valuation ring $F$; since $\bar{C} \cong \sigma$, we have $\xi(C)=$ $\xi(C[[t]]) \subseteq \xi(\bar{C}) \subseteq \xi(\bar{C}[[t]] \cap \widetilde{O}) \subseteq \xi(F)$; furthermore $\xi(F)$ is algebraic over $\xi(C[[t]])$ by $[1,(14.5)]$, and $\xi(\bar{C}) \cong \bar{C}$ is algebraically closed; thus $\xi(\bar{C}[[t]] \cap \mho)=\xi(F)$. On the other hand we have clearly $w(C[[t]])=$ $w(\bar{C}[[t]] \cap \widetilde{O})$. Thus by 3.2, $\bar{C}((t)) \cap \widetilde{O}$ is the inertia field of $F$ over $K^{*}$.

Step 2. Let $N_{\alpha}$ be a finite normal extension of $K$ contained in $N$. Let $N_{\alpha}^{*}$ be the completion of $N_{\alpha}$ contained in $N^{*}$. Then $\bar{C}((t)) \cap$ $N_{\alpha}^{*}$ is the inertia field of $B^{*} \cap N_{\alpha}^{*}$ over $K^{*}$ and $\bar{C}[[t]] \cap N_{\alpha}^{*}=\left(B^{*} \cap N_{\alpha}^{*}\right) \cap$ $\left(\bar{C}((t)) \cap N_{\alpha}^{*}\right)$.

Proof. $N_{\alpha}^{*}$ is a finite normal extension of $K^{*}$ [4, Corollary 4, p. 41]; hence $N_{\alpha}^{*} \cong \mho$. $B^{*} \cap N_{\alpha}^{*}$ is a valuation ring of $N_{\alpha}^{*}$ which lies over $A^{*}$; hence it has to be equal to $F \cap N_{\alpha}^{*}$. Now, the inertia field of $F \cap N_{\alpha}^{*}$ over $K^{*}$ is equal to the intersection of the inertia field of $F$ over $K^{*}$ with $N_{\alpha}^{*}[1,(19.10)]$, i.e., is equal to $(\bar{C}((t)) \cap \delta) \cap N_{\alpha}^{*}=$ 
$\bar{C}((t)) \cap N_{\alpha}^{*}$. Finally, $\bar{C}[[t]] \cap N_{\alpha}^{*}$ is a valuation ring of $\bar{C}((t)) \cap N_{\alpha}^{*}$ which lies over $A^{*}$, thus it has to lie under $B^{*} \cap N_{\alpha}^{*}$, i.e., we need to have $\left.\bar{C}[[t]] \cap N_{\alpha}^{*}=\left(B^{*} \cap N_{\alpha}^{*}\right) \cap \bar{C}((t)) \cap N_{\alpha}^{*}\right)$.

Step 3. $\bar{C}((t)) \cap N_{\alpha}$ is the inertia field of $B \cap N_{\alpha}$ over $K$ and $\bar{C}[[t]] \cap N_{\alpha}=\left(B \cap N_{\alpha}\right) \cap\left(\bar{C}((t)) \cap N_{\alpha}\right)$.

Proof. $B \cap N_{\alpha} \cap \bar{C}((t)) \cong B^{*} \cap N_{\alpha}^{*} \cap \bar{C}((t))=\bar{C}[[t]] \cap N_{\alpha}^{*}$ by Step 2; then, being contained in $\bar{C}((t)) \cap N_{\alpha}, B \cap N_{\alpha} \cap \bar{C}((t))$ has also to be contained in $\bar{C}[[t]] \cap N_{\alpha}$; being a rank-1 valuation ring, $B \cap N_{\alpha} \cap \bar{C}((t))$ has to be equal to $\bar{C}[[t]] \cap N_{\alpha}$.

Now, if we still call $w$ the valuation of $\sigma$ corresponding to $F$, we have $w(K) \cong w\left(\bar{C}((t)) \cap N_{\alpha}\right) \cong w\left(\bar{C}((t)) \cap N_{\alpha}^{*}\right)$; but $w\left(K^{*}\right)=w(\bar{C}((t)) \cap$ $N_{\alpha}^{*}$ ) by Step 2, and $w(K)=w\left(K^{*}\right)$ because, by [5, (1-7-5), p. 31], the completion is an immediate extension; hence $w(K)=w\left(\bar{C}((t)) \cap N_{\alpha}\right)$, and $\bar{C}((t)) \cap N_{\alpha} \subseteq K^{T}\left(B \cap N_{\alpha} / K\right)$ by 3.3. Then, $\bar{C}((t)) \cap N_{\alpha}=K^{T}\left(B \cap N_{\alpha} / K\right)$, because if not, the completion $L$ of $K^{T}\left(B \cap N_{\alpha} / K\right)$ contained in $N_{\alpha}^{*}$ would be such that $L \nsubseteq C((t)) \cap N_{\alpha}^{*}$ and $e\left(B^{*} \cap L / K^{*}\right)=1$, which is impossible by 3.3 , since $\bar{C}((t)) \cap N_{\alpha}^{*}$ is the inertia field of $B^{*} \cap N_{\alpha}^{*}$ over $K^{*}$ by Step 2 .

Step 4. $\bar{C}((t)) \cap N$ is the inertia field of $B$ over $K$ and $\bar{C}[[t]] \cap N=$ $B \cap(\bar{C}((t)) \cap N)$.

Proof. Let $\left\{N_{\alpha} ; \alpha \in J\right\}$ be the set of all the finite normal subextensions of $N$ over $K$. Let us show that $K^{T}(B \mid K)=\bigcup_{\alpha \in J} K^{T}\left(B \cap N_{\alpha} \mid K\right)$. For any $\alpha \in J$, the homomorphism $\theta_{\alpha}^{T}: G^{T}(B \mid K) \rightarrow G^{T}\left(B \cap N_{\alpha} \mid K\right)$ defined by $\theta_{\alpha}^{T}(\rho)=\left.\rho\right|_{N_{\alpha}}=$ the restriction of $\rho$ to $N_{\alpha}$, is surjective [1, (19.7]]. Let $x \in K^{T}(B \mid K), N_{\alpha}$ a finite normal extension of $K$ containing $x$ and $\sigma \in G^{T}\left(B \cap N_{\alpha} \mid K\right)$; since $\theta_{\alpha}^{T}$ is surjective, there exists $\rho \in G^{T}(B \mid K)$ such that $\left.\rho\right|_{N_{\alpha}}=\sigma$, so that $\sigma(x)=\rho(x)=x$ and $x \in K^{T}\left(B \cap N_{\alpha} \mid K\right)$. Conversely, let $\alpha \in J$, and $x \in K^{T}\left(B \cap N_{\alpha} \mid K\right)$; for any $\rho \in G^{T}(B \mid K)$ we have $\left.\rho\right|_{N_{\alpha}} \in G^{T}\left(B \cap N_{\alpha} \mid K\right)$, so that $\rho(x)=\left.\rho\right|_{N_{\alpha}}(x)=x$ and $x \in K^{T}(B \mid K)$. Hence, $K^{T}(B \mid K)=\bigcup_{\alpha \in J} K^{T}\left(B \cap N_{\alpha} \mid K\right)=\bigcup_{\alpha \in J}\left(\bar{C}((t)) \cap N_{\alpha}\right)=\bar{C}((t)) \cap\left(\bigcup_{\alpha \in J} N_{\alpha}\right)=$ $\bar{C}((t)) \cap N$, and $B \cap K^{T}(B \mid K)=B \cap\left(\bigcup_{\alpha \in J} K^{T}\left(B \cap N_{\alpha} \mid K\right)\right)=\bigcup_{\alpha \in J}(B \cap$ $\left.K^{T}\left(B \cap N_{\alpha} \mid K\right)\right)=\bigcup_{\alpha \in J}\left(\bar{C}[[t]] \cap N_{\alpha}\right)=\bar{C}[[t]] \cap N$.

4. Extensions of the derivation in the algebraic closure of the quotient field.

Lemma 4.1. Let $A$ be a ring, $I$ a finitely generated ideal of $A$ such that $\bigcap_{n=0}^{\infty} I^{n}=(0), A^{*}$ the I-adic completion of A. Let $D: A \rightarrow$ $A^{*}$ be a map such that $D(x+y)=D(x)+D(y)$ and $D(x y)=x D(y)+$ $y D(x)$. Then, 
(i) $D$ can be extended to a derivation $D^{\prime}$ on $A^{*}$ by $D^{\prime}\left(\lim _{n} x_{n}\right)=$ $\lim _{n} D\left(x_{n}\right)$, where $\left\{x_{n}\right\}_{n \geqq 0}$ is a Cauchy sequence in $A$.

(ii) $D^{\prime}$ is the only derivation of $A^{*}$ that extends $D$.

Proof. (i) Let $\left\{x_{n}\right\}_{n \geqq 0}$ be a Cauchy sequence in $A$; for any positive integer $m$, there exists $q$ such that $r, s>q \Rightarrow x_{r}-x_{s} \in I^{m} ; x_{r}-x_{s} \in$ $I^{m} \Rightarrow x_{r}-x_{s}=\sum_{i} u_{i 1} \cdots u_{i m}$ with $u_{i j} \in I$, hence $D x_{r}-D x_{s}=D\left(x_{r}-x_{s}\right)=$ $\sum_{i} \sum_{j=1}^{m} u_{i j} \cdots u_{i(j-1)} D\left(u_{i 1}\right) u_{i(j+1)} \cdots u_{i m} \in\left(I A^{*}\right)^{m-1}$; then as $I$ is finitely generated, the topology of $A^{*}$ is the $\left(I A^{*}\right)$-adic topology [7, Corollary 1 , p. 257], and $\left\{D x_{n}\right\}_{n \geqq 0}$ is a Cauchy sequence in $A^{*}$; set $D^{\prime}\left(\lim _{n} x_{n}\right)=$ $\lim _{n} D\left(x_{n}\right)$. Defined that way, $D^{\prime}$ is a function of $A^{*}$ for if $\left\{z_{n}\right\}_{n \geqq 0}$ is another Cauchy sequence such that $\lim _{n} x_{n}=\lim _{n} z_{n}$, then for any positive integer $m$, there exists $q$ such that $n>q \Rightarrow\left(x_{n}-z_{n}\right) \in I^{m}$, so that $D\left(x_{n}\right)-D\left(z_{n}\right)=D\left(x_{n}-z_{n}\right) \in\left(I A^{*}\right)^{m-1}$, and $\lim _{n} D\left(x_{n}\right)=\lim _{n} D\left(z_{n}\right)$. Furthermore, $D^{\prime}$ is a derivation of $A^{*}$ for if $\left\{x_{n}\right\}_{n \geqq 0}$ and $\left\{z_{n}\right\}_{n \geqq 0}$ are two Cauchy sequences of $A$, then $\lim _{n} \mathrm{D}\left(x_{n}+z_{n}\right)=\lim _{n} D\left(x_{n}\right)+\lim _{n} D\left(z_{n}\right)$ and $\lim _{n} D\left(x_{n} \cdot z_{n}\right)=\lim _{n} x_{n} \cdot \lim _{n} D\left(z_{n}\right)+\lim _{n} D\left(x_{n}\right) \cdot \lim _{n} z_{n}$ since, for every $n$, we have $D\left(x_{n}+z_{n}\right)=D\left(x_{n}\right)+D\left(z_{n}\right)$ and $D\left(x_{n} \cdot z_{n}\right)=x_{n} \cdot D\left(z_{n}\right)+$ $D\left(x_{n}\right) \cdot z_{n}$. Finally, for any $y \in A$, we clearly have $D^{\prime}(y)=D(y)$.

(ii) Let $D^{\prime \prime}$ be a derivation of $A^{*}$ which extends $D$. Let $y$ be any element of $A^{*}$, and $\left\{x_{n}\right\}_{n \geq 0}$ a Cauchy sequence in $A$ such that $y=\lim _{n} x_{n}$; then, for any positive integer $m$, there exists $q$ such that $n>q \Rightarrow y-y_{n} \in\left(I A^{*}\right)^{m}$, so that $D^{\prime \prime}(y)-D\left(y_{n}\right)=D^{\prime \prime}(y)-D^{\prime \prime}\left(y_{n}\right)=$ $D^{\prime \prime}\left(y-y_{n}\right) \in\left(I A^{*}\right)^{m-1}$, and $D^{\prime \prime}(y)=\lim _{n} D\left(y_{n}\right)=D^{\prime}(y)$.

REMARK. In the case of $D$ being a derivation of $A$, the procedure used in the preceding lemma allows to extend $D$ to a derivation $D^{\prime}$ of $A^{*}$ even if $I$ is not finitely generated. To get the uniqueness property however, we again need $I$ to be finitely generated.

THEOREM 4.2. Let $A$ be a rank-1 discrete valuation ring containing the rational numbers with quotient field $K$; let $\Omega$ be an algebraic closure of $K$ and $D$ a derivation of $A$. Let $B$ be a valuation ring of $\Omega$ lying over $A$; let $V$ be a valuation ring contained in $\Omega$, lying over $A$ and unramified over $K$. Then,

(i ) $\left(K^{T}(B \mid K), B \cap K^{T}(B \mid K)\right)$ is a D-regular extension of $(K, A)$ contained in $(\Omega, B)$.

(ii) $(N, B \cap N)$ is D-regular for any field $N$ between $K$ and $K^{T}(B \mid K)$.

(iii) $D$ is regular on $V$.

Proof. (i) Let $\left(\Omega^{*}, B^{*}\right)$ be a completion of $(\Omega, B)$ and $\left(K^{*}, A^{*}\right)$ 
the completion of $(K, A)$ contained in $\left(\Omega^{*}, B^{*}\right)$; let $\sigma^{\prime}$ be an algebraic closure of $\Omega^{*}$ and $\widetilde{O}$ the algebraic closure of $K^{*}$ contained in $\sigma^{\prime}$. Let $t$ be a generator of the maximal ideal of $A$; let $C$ be a field of representatives of $A^{*}$, and $\bar{C}$ the algebraic closure of $C$ in $\delta$; of course we have $A^{*}=C[[t]]$ and $K^{*}=C((t))\left[7\right.$, Corollary, p. 307]. By 4.1, let $D^{\prime}$ be the unique derivation of $A^{*}$ which is an extension of $D$, and, as usual, call again $D^{\prime}$ its extension to $\delta$. For an element $y$ of $\bar{C}$, we have $D^{\prime}(y) \in \bar{C}[[t]]$; indeed, if $X^{n}+c_{1} X^{n-1}+\cdots+c_{n} \in C[X]$ is the minimal polynomial of $y$ over $C$, differentiating the equation $y^{n}+c_{1} y^{n-1}+\cdots+c_{n}=$ 0 , we get $\left(n y^{n-1}+c_{1}(n-1) y^{n-2}+\cdots+c_{n-1}\right) D^{\prime}(y)+\left(D\left(c_{1}\right) y^{n-1}+\cdots+\right.$ $\left.D\left(c_{n}\right)\right)=0$; the first factor of the first term is an element of $\bar{C}$, different from zero since $y$ is separable over $C$; the second term is an element of $\bar{C}[[t]]$; thus $D^{\prime}(y) \in \bar{C}[[t]]$. We also have $D^{\prime}(t) \in \bar{C}[[t]]$, so that the restriction $D^{\prime \prime}$ of $D^{\prime}$ to $\bar{C}[t]$ is a function with values in $\bar{C}[[t]]$ which satisfies the properties $D^{\prime \prime}(x+z)=D^{\prime \prime}(x)+D^{\prime \prime}(z)$ and $D^{\prime \prime}(x z)=x D^{\prime \prime}(z)+z D^{\prime \prime}(x)$; furthermore, $\bar{C}[[t]]$ is the $(t)$-adic completion of $\bar{C}[t]$; thus, by $4.1, D^{\prime \prime}$ can be extended to a derivation of $\bar{C}[[t]]$, which we call $D^{\prime \prime}$ again, by $D^{\prime \prime}\left(\sum_{i=0}^{\infty} d_{i} t^{i}\right)=\sum_{i=0}^{\infty} D^{\prime \prime}\left(d_{i} t^{i}\right)=$ $\sum_{i=0}^{\infty} D^{\prime}\left(d_{i} t^{i}\right)$. As $C[[t]]$ is the completion of $C[t]$ for the $(t)$-adic topology, by 4.1 also, we know that for an element $\sum_{i=0}^{\infty} c_{i} t^{i}$ of $C[[t]]$ we must have $D^{\prime}\left(\sum_{i=0}^{\infty} c_{i} t^{i}\right)=\sum_{i=0}^{\infty} D^{\prime}\left(c_{i} t^{i}\right)$, so that $D^{\prime}=D^{\prime \prime}$ on $A^{*}=$ $C[[t]]$; thus $D=D^{\prime \prime}$ on $A$, hence also on $K$. But we can even see that $D=D^{\prime \prime}$ on $\bar{C}((t)) \cap \Omega$; indeed, if $X^{m}+k_{1} X^{m-1}+\cdots+k_{m} \in K[X]$ is the minimal polynomial over $K$ of an element $z$ of $\bar{C}((t)) \cap \Omega$, we have $z^{m}+k_{1} z^{m-1}+\cdots k_{m}=0$, thus $D(z)=\left[D\left(k_{1}\right) z^{m-1}+\cdots+D\left(k_{m}\right)\right] \times$ $\left[m z^{m-1}+\cdots+k_{m-1}\right]^{-1}=\left[D^{\prime \prime}\left(k_{1}\right) z^{m-1}+\cdots+D^{\prime \prime}\left(k_{m}\right)\right]\left[m z^{m-1}+\cdots+k_{m-1}\right]^{-1}=$ $D^{\prime \prime}(z)$. Then, since $D$ is regular on $\Omega$, since $D^{\prime \prime}$ is regular on $\bar{C}[[t]]$, and since $D=D^{\prime \prime}$ on $\bar{C}((t)) \cap \Omega$, we get that $(\bar{C}((t)) \cap \Omega, \bar{C}[[t]] \cap \Omega)$ is $D$-regular; but by 3.5 we know that $\bar{C}((t)) \cap \Omega=K^{T}(B \mid K)$ and $\bar{C}[[t]] \cap$ $\Omega=B \cap K^{T}(B \mid K)$; thus $\left(K^{T}(B \mid K), B \cap K^{T}(B \mid K)\right)$ is $D$-regular.

(ii) Let $N$ be any field between $K$ and $K^{T}(B \mid K)$. $D$ is regular on $N$ and is regular on $B \cap K^{T}(B \mid K)$; thus $D$ is regular on ( $B \cap$ $\left.K^{T}(B \mid K)\right) \cap N=B \cap N$.

(iii) Let $B^{\prime}$ be a valuation ring of $\Omega$ lying over $V$; by 3.4 we have $V \subseteq K^{T}\left(B^{\prime} \mid K\right)$, so that $D$ is regular on $V$.

THEOREM 4.3. Let $A$ be a D-simple valuation ring with quotient field $K$; let $\Omega$ be an algebraic closure of $K$, and $B$ a valuation ring of $\Omega$ lying over $A$. Then, $\left(K^{T}(B \mid K), B \cap K^{T}(B \mid K)\right)$ is the biggest $D$ regular extension of $(K, A)$ contained in $(\Omega, B)$.

Proof. Being $D$-simple, $A$ contains the rational numbers; thus, by 4.2 , we know that $\left(K^{T}(B \mid K), B \cap K^{T}(B \mid K)\right)$ is $D$-regular. Now let $(L, E)$ be a $D$-regular extension of $(K, A)$ contained in $(\Omega, B)$; of 
course $E$ is rank-1, and thus $B$ lies over $E$; also $E$ is $D$-simple by 1.1. If $t$ is a generator of the maximal ideal of $A$, then $t$ is also a generator of the maximal ideal $\mathfrak{M}_{E}$ of $E$; indeed, otherwise we would have $t \in \mathfrak{M}_{E}^{2}$, hence also $D(t) \in \mathfrak{M}_{E}$ which cannot be since $D(t)$ is a unit in $A$. Thus, the index of ramification of $E$ over $K$ is equal to 1 , and by $3.3(L, E) \subseteq\left(K^{T}(B \mid K), B \cap K^{T}(B \mid K)\right)$.

CoROLLARY 4.4. Let $R$ be a $D$-simple ring with quotient field $K$; let $\Omega$ be an algebraic closure of $K$. Let $V$ be a valuation ring which contains $R$ and is contained in $\Omega$; let $e(V \mid K)$ be its ramification index over $K$. Then, the following statements are equivalent:

(i) $D$ is regular on $V$.

(ii) $e(V \mid K)=1$ and $D$ is regular on $V \cap K$.

Proof. If $D$ is regular on $V$, then $D$ is regular on $V \cap K$ since $D$ is also regular on $K$. Furthermore, $V \cap K$ contains $R$ which is $D$-simple; thus, by 1.1, $V \cap K$ is $D$-simple and, as already noticed in the proof of 4.3, this implies that $e(V \mid K)=1$. Conversely, if $D$ is regular on $V \cap K$ and if $e(V \mid K)=1$ we know that $D$ is regular on $V$ by 4.2 .

\section{REFERENCES}

1. O. Endler, Valuation Theory, Hochschultexte, Springer Verlag, Berlin-HeidelbergNew York, 1972.

2. Y. Lequain, Differential simplicity and complete integral closure, Pacific J. Math., 36 (1971), 741-751.

3. A. Seidenberg, Derivations and integral closure, Pacific J. Math., 16 (1966), 167173.

4. J. P. Serre, Corps Locaux, Hermann, Paris, 1962.

5. E. Weiss, Algebraic Number Theory, McGraw-Hill Book Co., New York, 1963.

6. O. Zariski and P. Samuel, Commutative Algebra, v. 1, Van Nostrand Co., 1958.

7. Commutative Algebra, v. 2, Van Nostrand Co., 1960.

Received December 7, 1971.

Instituto de Matematica Pura e Aplicada

RUA LUIS DE LAMŌES 68

RIO DE JANEIRO 


\section{PACIFIC JOURNAL OF MATHEMATICS}

\section{EDITORS}

D. Gilbarg and J. Milgram Stanford University

Stanford, California 94305

R. A. Beaumont

University of Washington

Seattle, Washington 98105
J. DugundJI

Department of Mathematics

University of Southern California

Los Angeles, California 90007

RICHARD ARENS

University of California

Los Angeles, California 90024

ASSOCIATE EDITORS
E. F. BECKENBACH
B. H. NeumanN
F. WOLF
K. YoSHIDA

\section{SUPPORTING INSTITUTIONS}

UNIVERSITY OF BRITISH COLUMBIA

CALIFORNIA INSTITUTE OF TECHNOLOGY

UNIVERSITY OF CALIFORNIA

MONTANA STATE UNIVERSITY

UNIVERSITY OF NEVADA

NEW MEXICO STATE UNIVERSITY

OREGON STATE UNIVERSITY

UNIVERSITY OF OREGON

OSAKA UNIVERSITY
UNIVERSITY OF SOUTHERN CALIFORNIA

STANFORD UNIVERSITY

UNIVERSITY OF TOKYO

UNIVERSITY OF UTAH

WASHINGTON STATE UNIVERSITY

UNIVERSITY OF WASHINGTON

$\stackrel{*}{*} \stackrel{*}{*} \stackrel{*}{*}$ AMERICAN MATHEMATICAL SOCIETY

NAVAL WEAPONS CENTER 


\section{Pacific Journal of Mathematics}

\section{Vol. 46, No. $1 \quad$ November, 1973}

Allan Francis Abrahamse, Uniform integrability of derivatives on $\sigma$-lattices .......................................... 1

Ronald Alter and K. K. Kubota, The diophantine equation $x^{2}+D=p^{n} \ldots \ldots \quad 11$

Grahame Bennett, Some inclusion theorems for sequence spaces .......... 17

William Cutler, On extending isotopies ........................ 31

Robert Jay Daverman, Factored codimension one cells in Euclidean

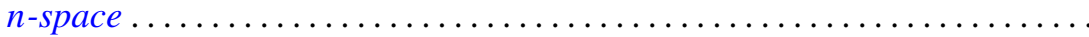

Patrick Barry Eberlein and Barrett O’Neill, Visibility manifolds ............ 45

M. Edelstein, Concerning dentability .......................... 111

Edward Graham Evans, Jr., Krull-Schmidt and cancellation over local

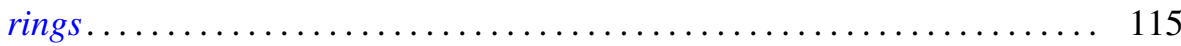

C. D. Feustel, A generalization of Kneser's conjecture ................ 123

Avner Friedman, Uniqueness for the Cauchy problem for degenerate parabolic equations .......................................... 131

David Golber, The cohomological description of a torus action ............ 149

Alain Goullet de Rugy, Un théorème du genre "Andô-Edwards" pour les

Fréchet ordonnés normaux..............................

Louise Hay, The class of recursively enumerable subsets of a recursively enumerable set ........................................

John Paul Helm, Albert Ronald da Silva Meyer and Paul Ruel Young, On orders of translations and enumerations...

Julien O. Hennefeld, A decomposition for $B(X)^{*}$ and unique Hahn-Banach

extensions

Gordon G. Johnson, Moment sequences in Hilbert space .

Thomas Rollin Kramer, A note on countably subparacompact spaces ...

Yves A. Lequain, Differential simplicity and extensions of a derivation ....

Peter Lorimer, A property of the groups Aut $\mathrm{PU}\left(3, q^{2}\right) \ldots$

225

Yasou Matsugu, The Levi problem for a product manifold.

231

John M.F. O'Connell, Real parts of uniform algebras ......

William Lindall Paschke, A factorable Banach algebra without bounded

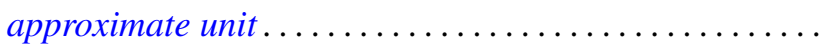

Ronald Joel Rudman, On the fundamental unit of a purely cubic field ....

Tsuan Wu Ting, Torsional rigidities in the elastic-plastic torsion of simply connected cylindrical bars .........................

Philip C. Tonne, Matrix representations for linear transformations on analytic sequences...................................

Jung-Hsien Tsai, On E-compact spaces and generalizations of perfect

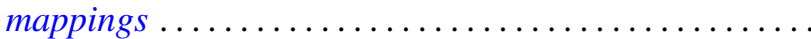

Alfons Van Daele, The upper envelope of invariant functionals majorized by an invariant weight. .. 